\title{
Heurísticas Contextuais para Portais Universitários
}

\author{
Andréa P. S. Pelogi ${ }^{1}$, Marcelo P. Guimarães ${ }^{2}$, Claudia G. N. Barsottini ${ }^{1}$ \\ ${ }^{1}$ Programa de Pós-Graduação em Gestão e Informática em Saúde do Departamento de Informática em \\ Saúde - Universidade Federal de São Paulo (UNIFESP). \\ ${ }^{2}$ Universidade Aberta do Brasil (UNIFESP)/Programa de mestrado em Ciência da Computação da \\ Faculdade Campo Limpo Paulista.
}

apelogi@gmail.com, marcelo.paiva@unifesp.br, claudia.barsottini@unifesp. $\underline{\mathrm{br}}$

\begin{abstract}
This study conducted a case study on the Portal of the Federal University of São Paulo through a Heuristic Evaluation to find usability problems. The research objective is to investigate whether contextual heuristics are appropriate and contribute to a more specific evaluation of interface usability of educational portals. We selected six experts. The result of the experiment showed that the use of specific heuristics was efficient in diagnosing usability problems in identifying 100 Unifesp Portal. From the results of the heuristic evaluation was also possible to introduce a new set of heuristics to capture contextual domain-specific aspects of educational portals.
\end{abstract}

Resumo. Os Portais Universitários são pontos de acesso único aos dados das Instituições de Ensino para os alunos, docentes, funcionários e demais interessados. Por isso, eles devem fornecer uma interface de fácil uso, com sequências simples e consistentes, e, além disso, mostrar claramente as alternativas existentes no processo da interação, deixando então o usuário seguro. Este trabalho visa averiguar as heurísticas propostas pelo Instituto Faber-Ludens para avaliar a usabilidade dos Portais Universitários. Para isso, avaliou-se o Portal da Universidade Federal de São Paulo, o que resultou na proposta de adaptação destas heurísticas e na proposta de novas heurísticas.

\section{Introdução}

A usabilidade é a propriedade que estabelece se o uso de um produto é simples e rapidamente aprendido, dificilmente esquecido, não gera erros operacionais, apresenta alto grau de contentamento para seus usuários e resolve com eficiência as tarefas para as quais ele foi elaborado. Para garantir a usabilidade de um site, deve-se dar atenção aos seus requisitos não funcionais, para afiançar que a informação dada ao usuário seja de qualidade (NIELSEN, 2000). Um produto dirigido para a usabilidade possui uma interface que é utilizada sem chamar atenção para si, permitindo que o usuário focalize sua energia somente no trabalho que almeja executar (NORMAN; DRAPER, 1986). Uma interface bem projetada deve ser guiada por metas de usabilidade e pela experiência do usuário. O objetivo de elaborar um produto interativo agradável, com boa estética está baseado na experiência que este produto proporcionará ao usuário, isto é, como o usuário se sentirá durante a interação. 
A avaliação de interface verifica a usabilidade e está associada às condições oferecidas aos usuários na utilização da funcionalidade da aplicação (NIELSEN; MOLICH, 1990). A literatura tem demonstrado que a Avaliação Heurística tem apresentado melhores resultados com menos esforço gasto no diagnóstico de problemas sérios quando comparado com outras técnicas (JEFFRIES et al., 1991). As heurísticas de usabilidade de software tem-se mostrado bastante úteis como instrumento de avaliação da qualidade da interação disponibilizada por software (COELHO; SANTORO, 2002). Este trabalho visa averiguar se as heurísticas desenvolvidas para avaliar Portais Universitários, que foram propostas pelo Instituto Faber-Ludens, são apropriadas. Para isto, aplicou-se essas heurísticas no Portal da Universidade Federal de São Paulo (Unifesp), o que resultou em sugestão de adaptação e em novas heurísticas, além da avaliação do portal.

O restante deste trabalho está organizado da seguinte forma: a seção 2 apresenta as heurísticas desenvolvidas pelo Instituto Faber-Ludens; a seção 3 os materiais e métodos utilizados neste trabalho; a seção 4 os resultados; a seção 5 mostra a discussão dos resultados; e, por fim, a seção 6 apresenta as conclusões.

\section{Heurísticas para Portais Educacionais}

A avaliação heurística tem como base um conjunto de princípios de usabilidade, que descrevem características desejáveis da interação e da interface chamadas por Nielsen de heurísticas (NIELSEN; HACKOS, 1993). As heurísticas de Nielsen têm como foco um sistema genérico, ou seja, não são destinadas a um contexto específico. Isso não impede que as heurísticas sejam utilizadas em qualquer interface. Uma avaliação também pode usar heurísticas voltadas para uma determinada categoria de produto, por meio de heurísticas derivadas do resultado de avaliações de produtos semelhantes (NIELSEN, 1995).

O Instituto Faber-Ludens criou as heurísticas específicas para Portais Educacionais (Quadro 1), que serão utilizadas nesse trabalho. Para elaborar essas heurísticas, foram analisados diversos portais educacionais considerados bons exemplos de interface (AMSTEL, 2011). Elas são as seguintes:

Quadro 1 - Heurísticas criadas pelo Faber-Ludens

\begin{tabular}{|l|l|}
\hline Heurística & Descrição \\
\hline $\begin{array}{l}\text { Atender os } \\
\text { interesses do } \\
\text { público }\end{array}$ & $\begin{array}{l}\text { Alunos, jornalistas, professores, pesquisadores e técnicos possuem interesses em } \\
\text { comum e interesses distintos. O portal deve atender, primeiramente, os interesses } \\
\text { comuns e, se possível, os interesses específicos de cada público; }\end{array}$ \\
\hline $\begin{array}{l}\text { Reforçar os } \\
\text { valores da } \\
\text { Universidade }\end{array}$ & $\begin{array}{l}\text { O portal deve estar alinhado com os valores que a Universidade cultiva, servindo não } \\
\text { só para difundi-los, mas também para atualizá-los; }\end{array}$ \\
\hline $\begin{array}{l}\text { Encontrar } \\
\text { informações sem } \\
\text { conhecer a } \\
\text { estrutura } \\
\text { organizacional }\end{array}$ & $\begin{array}{l}\text { Mesmo que o usuário desconheça quais são as fontes de informação da Universidade, } \\
\text { ele deve ter acesso às informações geradas por estas fontes. Deve haver maneiras de } \\
\text { encontrar informações por outros critérios além da estrutura organizacional; }\end{array}$ \\
\hline $\begin{array}{l}\text { Consistência na } \\
\text { navegação entre } \\
\text { divisões } \\
\text { organizacionais }\end{array}$ & $\begin{array}{l}\text { As Universidades costumam ter muitas subdivisões organizacionais e subsites para } \\
\text { cada uma delas. A navegação entre estes subsites deve manter os padrões mínimos de } \\
\text { identificação da Universidade e das divisões superiores, de modo a permitir imersão } \\
\text { no conteúdo sem se perder na estrutura organizacional; }\end{array}$ \\
\hline
\end{tabular}




\begin{tabular}{|c|c|}
\hline $\begin{array}{l}\text { Buscar por } \\
\text { informações } \\
\text { específicas }\end{array}$ & $\begin{array}{l}\text { Ferramentas de busca são essenciais para Portais com grande quantidade de } \\
\text { conteúdo, porém, esta deve estar devidamente projetada para exibir resultados } \\
\text { relevantes em um formato claro; }\end{array}$ \\
\hline $\begin{array}{l}\text { Auxiliar o aluno } \\
\text { potencial a } \\
\text { escolher seu curso }\end{array}$ & $\begin{array}{l}\text { O portal Web é frequentemente o primeiro contato do aspirante a aluno da } \\
\text { Universidade. Ele usa o portal para comparar os cursos que a Universidade oferece, } \\
\text { bem como para compará-los com cursos que outras Universidades oferecem. O portal } \\
\text { pode contribuir também para a orientação vocacional dos candidatos; }\end{array}$ \\
\hline $\begin{array}{l}\text { Explicar } \\
\text { claramente como } \\
\text { entrar na } \\
\text { Universidade }\end{array}$ & $\begin{array}{l}\text { Os processos seletivos e seus critérios devem estar bem definidos. O apoio que a } \\
\text { Universidade oferece para a manutenção dos aprovados pode estar associados à estas } \\
\text { informações; }\end{array}$ \\
\hline $\begin{array}{l}\text { Acesso à produção } \\
\text { científica e } \\
\text { inovações } \\
\text { tecnológicas }\end{array}$ & $\begin{array}{l}\text { O portal Web é uma forma econômica e eficiente para difundir a produção de } \\
\text { conhecimento dentro da Universidade. Além de servir pesquisadores de outras } \\
\text { instituições, o portal pode apresentar conhecimentos em um formato para leigos; }\end{array}$ \\
\hline $\begin{array}{l}\text { Reduzir a } \\
\text { burocracia }\end{array}$ & $\begin{array}{l}\text { Certos procedimentos burocráticos em Universidades envolvem conhecimentos que } \\
\text { somente as subdivisões responsáveis dominam. Uma vez que o usuário tem um } \\
\text { objetivo determinado, ele deve poder encontrar estas informações sem dificuldades. } \\
\text { O portal pode também oferecer serviços e atalhos para procedimentos de rotina; }\end{array}$ \\
\hline $\begin{array}{l}\text { Serviços à } \\
\text { comunidade }\end{array}$ & $\begin{array}{l}\text { O portal deve apresentar os serviços oferecidos à comunidade interna e externa à } \\
\text { Universidade de maneira clara, incluindo informações fundamentais sobre } \\
\text { benefícios, local, requisitos para uso dos serviços e quaisquer outras informações } \\
\text { necessárias aos usuários dos serviços; }\end{array}$ \\
\hline $\begin{array}{l}\text { Novos negócios, } \\
\text { projetos e } \\
\text { parcerias }\end{array}$ & $\begin{array}{l}\text { Novos negócios, projetos e parcerias devem ser estimulados pelo portal. O portal } \\
\text { deve ampliar as oportunidades de realização de novas parcerias, bem como, exibir as } \\
\text { áreas de interesse da Universidade perante as organizações; }\end{array}$ \\
\hline $\begin{array}{l}\text { Comunidade } \\
\text { Online }\end{array}$ & $\begin{array}{l}\text { O portal deve apoiar a criação de comunidades online por meio da criação de uma } \\
\text { estrutura adequada, bem como, integração com outras plataformas colaborativas } \\
\text { externas; }\end{array}$ \\
\hline $\begin{array}{l}\text { Relevância das } \\
\text { informações }\end{array}$ & $\begin{array}{l}\text { Quanto mais informação exibida, maior a dificuldade do usuário para distingui-las e } \\
\text { compreendê-las. As informações exibidas devem estar relacionadas com o contexto } \\
\text { do usuário. Informações que não tem relação entre si devem estar distantes ou } \\
\text { ausentes; }\end{array}$ \\
\hline $\begin{array}{l}\text { Responsabilidade } \\
\text { pela informação }\end{array}$ & $\begin{array}{l}\text { Citar a fonte de cada informação publicada dá credibilidade ao portal e permite } \\
\text { aprofundamento, caso o usuário deseje entrar em contato com o órgão responsável. } \\
\text { Além disso, gera uma cobrança saudável pela manutenção das informações } \\
\text { atualizadas. }\end{array}$ \\
\hline
\end{tabular}

\section{Material e Métodos}

No Portal atual da Unifesp, os espaços estão distribuídos com o objetivo de potencializar a apropriação dos produtos e serviços disponibilizados pelos Campi, Departamentos, Disciplinas e Setores por meio do oferecimento de serviços de ensino e pesquisa. A estrutura inicial do portal (Figura 1) apresenta as seguintes funcionalidades no menu vertical na área direita: "Cursos", "Estrutura do Campi", "Fundação", "Informações", “Órgãos e Associações”. No menu horizontal apresentam-se as opções: "Unifesp", "Reitoria", "Administração", "Graduação", "Extensão", "Pós-Graduação", "Planejamento" e "Assuntos Estudantis". Essas funcionalidades visam organizar o conteúdo produzido e os serviços que serão acessados pelos usuários do Portal. $\mathrm{Na}$ área 
central são disponibilizados os destaques, as notícias e a busca do Portal. Na área lateral direita foram colocados os ícones dos links mais buscados no campo busca.

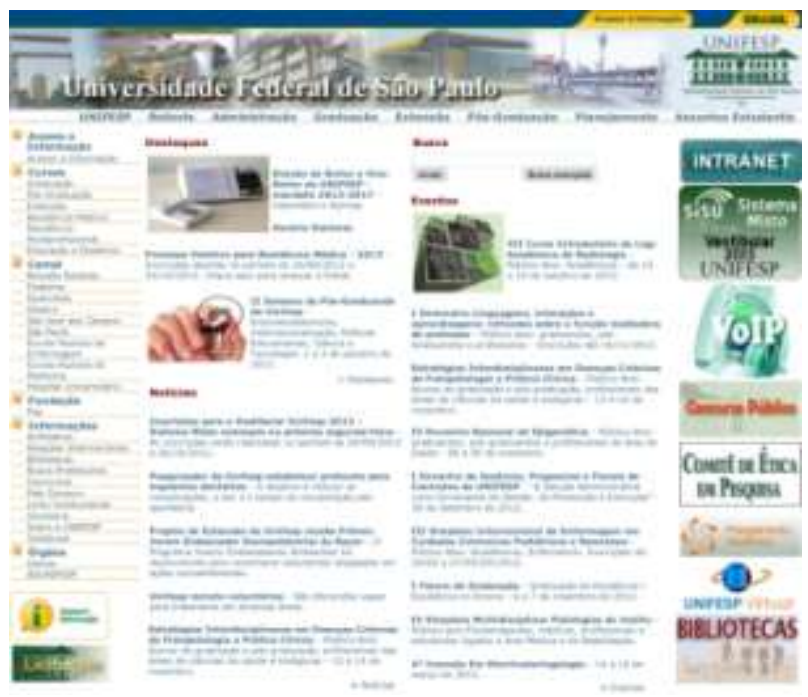

Figura 1 - Versão atual do Portal da Universidade Federal de São Paulo. URL: http://www.unifesp.br - Data de Acesso: janeiro de 2013.

O procedimento para a avaliação consistiu de três fases descritas no Quadro 2. O resultado do trabalho desenvolvido nestas 3 fases foi a confecção de um relatório com a lista de problemas de usabilidade encontrados na interface do sistema.

Quadro 2 - Fases da Avaliação Heurística do Portal Unifesp

\begin{tabular}{|l|l|l|}
\hline Fases & Descrição & Tempo \\
\hline Pré-avaliação & $\begin{array}{l}\text { Início da Apresentação do Portal: 10:00h } \\
\text { Preenchimento do Termo de Consentimento Livre e Esclarecido }\end{array}$ & $20 \mathrm{~min}$ \\
\hline Avaliação & $\begin{array}{l}\text { Início da avaliação: 10:20h. } \\
\text { Término da avaliação: } 11: 10 \mathrm{~h} .\end{array}$ & $50 \mathrm{~min}$ \\
\hline $\begin{array}{l}\text { Discussão } \\
\text { entre os } \\
\text { avaliadores }\end{array}$ & $\begin{array}{l}\text { Início da Discussão: } 11 \mathrm{~h} 10 \mathrm{~min} . \\
\text { Fim da Discussão: } 12 \mathrm{~h} 00 \mathrm{~min} .\end{array}$ & $50 \mathrm{~min}$ \\
\hline & $\begin{array}{l}\text { Os avaliadores discutiram pontos comuns e pontos discordantes e } \\
\text { classificaram a severidade dos problemas }\end{array}$ & $120 \mathrm{~min}$ \\
\hline
\end{tabular}

O perfil dos avaliadores está descrito no Quadro 3.

Quadro 3 - Perfil dos Avaliadores que avaliaram o Portal Unifesp

\begin{tabular}{|l|c|c|c|c|c|c|}
\hline \multirow{2}{*}{ Dados } & \multicolumn{7}{|c|}{ Avaliadores } \\
\cline { 2 - 7 } Sexo & $\mathbf{1}$ & $\mathbf{2}$ & $\mathbf{3}$ & $\mathbf{4}$ & $\mathbf{5}$ & $\mathbf{6}$ \\
\hline Idade & Homem & Homem & Homem & $\begin{array}{c}\text { Home } \\
\mathrm{m}\end{array}$ & Homem & Homem \\
\hline Formação & 35 & 23 & 34 & 47 & 25 & 42 \\
\hline $\begin{array}{l}\text { Participou da equipe de } \\
\text { desenvolvimento }\end{array}$ & Mestre & Especialista & Graduado & Mestre & Mestre & Doutor \\
\hline Especialista do domínio & Sim & Não & Não & Não & Não & Não \\
\hline Especialista em usabilidade & Não & Sim & Não & Sim & Sim & Sim \\
\hline
\end{tabular}

Os avaliadores foram convidados a participar da pesquisa por um convite enviado por email um mês antes da avaliação. Com a confirmação de aceitação, eles receberam uma semana antes da data marcada para a avaliação: email com o objetivo do 
projeto; breve resumo sobre o que é uma avaliação heurística; explanação sobre a categorização dos graus de severidade; lista de heurísticas para portais universitários; e instruções do experimento, incluindo as orientações sobre o plug-in notableapp (STEWART, 2012), que permite selecionar uma parte da tela e fazer comentários sobre a respectiva área.

Um observador ficou presente durante a sessão de avaliação. Foi solicitado aos avaliadores acessar o portal procurando a orientação de uso do mesmo, através dos menus e ícones. Foi feita uma recomendação para que o acesso visasse a exploração a fim de verificar os problemas de usabilidade de forma individual. Todos os avaliadores completaram uma sessão de uso do sistema sem grandes dificuldades, incluindo os dois sem conhecimento especializado do domínio. Coube também aos avaliadores classificar o grau de severidade dos problemas de usabilidade detectados.

$\mathrm{Na}$ fase de discussão, os avaliadores e o observador discutiram as características gerais da inspeção de usabilidade dos resultados individuais encontrados, o que resultou em um único relatório. Foram discutidos e enumerados os problemas mais graves que sugerem necessidade de um novo redesenho.

\section{Resultados}

Os avaliadores em consenso associaram cada problema encontrado uma descrição, a respectiva heurística afetada, o grau de severidade e a localização. No entanto, alguns problemas não se relacionavam com nenhuma heurística (Quadro 4).

Quadro 4 - Problemas encontrados e não relacionados a nenhuma heurística

\begin{tabular}{|l|c|}
\hline Problemas não relacionados a nenhuma heurística & Tipo de Heurística \\
\hline Adicionar outros critérios importantes para avaliação do portal. & Outros critérios \\
\hline Layout fora de moda & Layout \\
\hline Layout inadequado sem descriminação de cores, posição & Layout \\
\hline Menu com muitas grafias e linhas desnecessárias & Layout \\
\hline Muito espaço sem utilização & Layout \\
\hline Não segue layout consolidados de portais de notícias populares & Acessibilidade \\
\hline Portal não atende especificidade de aumento de letra & Dispositivo móvel \\
\hline Portal não atende especificidade de dispositivo de acesso do consumidor & \\
\hline Total = 8 problemas sem heurísticas & \\
\hline
\end{tabular}

Após a análise dos problemas, pode-se concluir que as heurísticas mais desrespeitadas foram a "Interesse do Público", com 22 problemas encontrados, depois "Consistência entre as divisões" e "Busca por informação específica" que tiveram 11 problemas encontrados, cada uma. A heurística com menos problemas encontrados foi a "Reduzindo a burocracia" que foi classificada em problemas. Todas as heurísticas foram selecionadas pelo menos uma vez. A Tabela 1 apresenta a relação de heurísticas e os graus de severidade (NIELSEN, 1994), que foram atribuídos a cada uma delas. 
Tabela 1 - Heurísticas x Severidade

\begin{tabular}{|c|c|c|c|c|c|}
\hline Heurísticas & Sem Importância & Cosmético & Simples & Grave & Catastrófico \\
\hline Interesse do público & & $3(3 \%)$ & $13(13 \%)$ & $6(6 \%)$ & \\
\hline Consistência & & $1(1 \%)$ & $5(5 \%)$ & $4(4 \%)$ & $1(1 \%)$ \\
\hline Busca & $1(1 \%)$ & $1(1 \%)$ & $7(7 \%)$ & $1(1 \%)$ & $1(1 \%)$ \\
\hline Estrutura organizacional & & $1(1 \%)$ & $3(3 \%)$ & $4(4 \%)$ & \\
\hline Valores & $1(1 \%)$ & $1(1 \%)$ & $2(2 \%)$ & $3(3 \%)$ & \\
\hline Responsabilidade & & $1(1 \%)$ & $5(5 \%)$ & $1(1 \%)$ & \\
\hline Ingresso & & $2(2 \%)$ & & & $4(4 \%)$ \\
\hline Serviços & & $1(1 \%)$ & $3(3 \%)$ & $2(2 \%)$ & \\
\hline Comunidade online & & $2(2 \%)$ & $2(2 \%)$ & & $1(1 \%)$ \\
\hline Relevância & & & $3(3 \%)$ & $2(2 \%)$ & \\
\hline Projetos & & & $1(1 \%)$ & $3(3 \%)$ & \\
\hline Cursos & $1(1 \%)$ & $1(1 \%)$ & & $1(1 \%)$ & \\
\hline Produção Científica & & & $1(1 \%)$ & $2(2 \%)$ & \\
\hline Burocracia & & & & $2(2 \%)$ & \\
\hline Total & $3(3 \%)$ & $14(14 \%)$ & $45(45 \%)$ & $31(31 \%)$ & $7(7 \%)$ \\
\hline
\end{tabular}

Assim, pode-se concluir que a área do Portal com maior número de problemas de usabilidade é a "Área geral" (12\%) seguida por "Ícones a Direita", "Notícias e Eventos" (10\%), Busca (9\%) e Menu Vertical (8\%). Cabe ressaltar que algumas dessas áreas analisadas não existem no Portal Unifesp. Contudo, podem entrar como sugestão para o projeto de desenvolvimento de um novo Portal: Canais, Produção Científica, Comunidades online, Visão da Universidade, Breadcrumb, Ingresso, Serviços e Origem da Informação. Embora a avaliação heurística não substitua os testes com usuários, permitiu uma avaliação global da interface e foi eficaz para identificação dos problemas de usabilidade.

\section{Discussão}

Durante a avaliação do Portal da Unifesp buscou-se verificar os alcances dos procedimentos existentes e contribuir para identificar critérios específicos de análise de tais portais. Também se procurou agregar fundamentos para futura elaboração de método heurístico específico para interfaces desta natureza.

A avaliação heurística, utilizando as heurísticas criadas por Faber Ludens, mostrou-se eficiente no diagnóstico de problemas de usabilidade, identificando um total de 108 problemas no estudo de caso. Dos 108 problemas, foram descobertos oito que não se relacionaram a nenhuma heurística. Desses oito, cinco eram relacionados a problemas de layout, dois relacionados a problemas de acessibilidade e um problema relacionado à falta de padrões para hierarquização de menus. Os oitos problemas não relacionados às heurísticas para Portais Universitários foram considerados de forma separada no estudo.

Os 100 problemas encontrados e relacionados as quatorze heurísticas específicas foram classificados da seguinte maneira: $22 \%$ problemas de usabilidade relacionados "interesse do público", $11 \%$ a "busca pela informação", $11 \%$ a "consistência da navegação", 8\% a "estrutura organizacional", 7\% a "valores da universidade", 7\% a "responsabilidade pela informação", 6\% a "ingresso na universidade", 6\% a "serviços", 5\% a "comunidade online", 5\% a "relevância das informações", 4\% a "projetos e parcerias", $3 \%$ a "cursos", $3 \%$ a "produção científica" e $2 \%$ a heurística "burocracia". 
Apenas dois avaliadores possuíam conhecimentos avançados de usabilidade e quatro nunca haviam participado de uma avaliação heurística anteriormente. A pouca experiência dos avaliadores em usabilidade pode ter sido um dos pontos fracos da pesquisa. No entanto, foi constatado o diagnóstico de um grande número de problemas de severidade grave $(31 \%)$ ou catastrófica $(7 \%)$, mesmo por aqueles com pouca experiência na área de usabilidade. Isto indica a alta visibilidade de tais problemas na interface analisada. A constatação desses $38 \%$ de problemas graves ou catastróficos reforça a importância dos problemas diagnosticados pela avaliação heurística.

ANDRADE (2007) fez uma comparação entre a avaliação heurística e o teste de usabilidade. $\mathrm{O}$ uso do teste de usabilidade como método complementar à avaliação heurística, não acarretou um grande número de novos problemas de usabilidade (ANDRADE, 2007). Santinho (2001) também comparou os dois métodos. O estudo revelou que a avaliação heurística mostrou-se mais eficaz na identificação de maior número de problemas do que o teste de usabilidade (SANTINHO, 2001). Esses resultados não desqualificam o teste de usuários. No entanto, é preciso elaborar outros estudos para comprovar a complementaridade e a convergência entre os dois métodos.

A metodologia aplicada apresenta algumas limitações:

- As avaliações realizadas não têm o poder de modificar a situação encontrada, servindo apenas como um diagnóstico;

- A seleção de avaliadores especialistas em usabilidade aumenta as chances de detecção de problemas na interface. A qualidade e quantidade de problemas diagnosticados pelos avaliadores selecionados foram adequadas. Contudo, selecionar avaliadores especialistas em usabilidade é complexo pela falta de oferta desse profissional no Brasil. Considera-se, no entanto, que não comprometem o desenvolvimento da pesquisa, nem tem impacto essencial na qualidade dos resultados.

GONZALES et al. (2008) também avaliaram a usabilidade de Portais Universitários de língua espanhola. Foram analisados fatores como "Design Gráfico" (28\%), "Área de Navegação" (28\%), "Conteúdo" (20\%), "Busca" (24\%). No Portal Unifesp, o item "Busca" obteve 11\% das anotações de problemas de usabilidade. Os avaliadores registraram que ferramentas de busca são essenciais para Portais com grande quantidade de conteúdo, porém, esta deve estar devidamente projetada para exibir resultados relevantes em um formato claro. Outra anotação dos avaliadores foi de que a caixa de busca está em uma região que não facilita encontrá-la uma vez que está junto às notícias e outras informações. Deveria estar com mais destaque e no cabeçalho como diversos portais utilizam. Também no Portal da Unifesp, a área de navegação recebeu críticas (Menu Vertical (8\%) e Menu Horizontal (5\%)). Os avaliadores consideram que o menu deve ser único e o excesso de informações prejudica a clareza da informação (GONZÁLEZ et al., 2008). Também anotaram que o menu deve ser mais intuitivo e evitar a poluição visual, demonstrar a estrutura organizacional impecável e hierarquicamente correta.

$\mathrm{Na}$ avaliação heurística realizada por KOSTARAS \& XENOS (2007) no site da Hellenic Open University da Grécia, as 10 heurísticas de Nielsen foram aplicadas e 38 problemas de usabilidade foram encontrados. Desses, 32\% foram problemas associados à heurística "consistência e padrões". Assim como no Portal Unifesp, a avaliação revelou que a estrutura do menu não promove a satisfação do usuário quando ele busca 
por informações. Também foram encontrados $29 \%$ de problemas associados à heurística "estética e design" que apresenta como maior problema de usabilidade o fato do site ser grego e não ter tradução para nenhuma outra língua (KOSTARAS; XENOS, 2007). Do mesmo modo, no Portal Unifesp, problemas relacionados com "estética e design" também foram relacionados durante a avaliação. Foram eles: estética geral, ícones laterais, menus, rodapé, logo da universidade e cabeçalho foram relatados e somam $44 \%$ dos problemas de estética e design encontrados.

DAHER \& ELKABANI (2012) investigaram os fatores que afetam a usabilidade de Portais Universitários de nove universidades do Líbano (DAHER; ELKABANI, 2012). Nesse estudo também foram selecionados avaliadores especialistas de domínio (alunos e professores) que classificaram o nível de usabilidade utilizando a seguinte escala: "sempre usável", "às vezes usável", "raramente usável", "nunca usável" e "não se aplica". Como resultado, o trabalho apresenta a lista de serviços oferecidos pelas universidades e o seu nível de usabilidade. Os piores resultados "raramente usável" ou "nunca usável" foram obtidos na página dos cursos, na página de notícias e na biblioteca digital. Já no Portal Unifesp também receberam críticas sobre a qualidade e a disposição das notícias com $10 \%$ de anotações e a dificuldade de se encontrar os cursos na busca e em todo o portal com 3\%. Também há que se considerar a heurística que anotou dificuldades dos usuários em encontrar a forma de ingresso na Universidade com $6 \%$ das ocorrências.

Observou-se que grande parte dos problemas identificados é de fácil solução, não demandando modificações estruturais. No entanto, tais correções colocam-se como realmente essenciais, uma vez que a existência de tais problemas influencia diretamente na satisfação do usuário. Ao mesmo tempo, outros problemas de usabilidade encontrados precisam de modificações profundas, sendo inclusive necessário um reprojeto da interface e também uma revisão conceitos aplicados.

Cabe ressaltar que o intuito não é bloquear a criatividade dos desenhistas de interface web e sim nortear o seu trabalho para que possam exercer sua capacidade criadora considerando os benefícios que a construção de interfaces baseadas em princípios de usabilidade pode apresentar aos usuários.

O maior público do Portal é composto por alunos, professores/pesquisadores e funcionários. É preciso que o Portal tenha canais específicos para atender a demanda de cada público. O usuário precisa encontrar resultados relevantes em um formato claro quando utiliza a busca. Além disso, é preciso organizar a estrutura dele para que o usuário tenha acesso às divisões internas de forma mais clara.

Como relatado pelos avaliadores, algumas heurísticas podem interferir em outras. Sugere-se então que sejam compactadas e agrupadas como mostra o Quadro 5.

Quadro 5 - Adaptações das Heurísticas de Faber Ludens

\begin{tabular}{|l|}
\hline 1. Atender os interesses do público. \\
\hline 2. Reforçar os valores da Universidade. \\
\hline 3. Buscar informações sem burocracia. \\
\hline 4. Consistência na navegação entre divisões organizacionais. \\
\hline 5. Informações para Ingresso na Universidade. \\
\hline 6. Acesso à produção científica e inovações tecnológicas. \\
\hline 7. Serviços à comunidade. \\
\hline 8. Novos negócios, projetos e parcerias. \\
\hline
\end{tabular}


9. Comunidade Online.

10. Relevância e Inconsistência das informações.

11. Responsabilidade pela informação.

Com base nos resultados, observou-se também, a necessidade e a possibilidade de acrescentar novas heurísticas (Quadro 6):

\section{Quadro 6 - Novas heurísticas.}

\begin{tabular}{|l|l|}
\hline 12. Padrão Visual & $\begin{array}{l}\text { O site deve possuir cores uniformes nas páginas combinando com a imagem } \\
\text { corporativa da universidade e oferecer uma interface limpa, sem ruído visual e } \\
\text { com um uso correto do espaço. O texto deve ter um design simples, com } \\
\text { bastante contraste entre o fundo e o texto, limitando o tipo de fonte. }\end{array}$ \\
\hline 13. Imagens & $\begin{array}{l}\text { As imagens devem estar rotuladas e possuir a descrição quando se passa o } \\
\text { mouse sobre elas. Os elementos animados não podem dificultar o acesso. A } \\
\text { resolução das imagens deve ser adequada para sua visualização correta. }\end{array}$ \\
\hline 14. Atualização do site & As notícias devem estar devidamente atualizadas e ter data de publicação. \\
\hline 15. Internacionalização & $\begin{array}{l}\text { O site deve oferecer opção de vários idiomas. A informação acadêmica deve } \\
\text { ficar disponível para as diferentes línguas. }\end{array}$ \\
\hline
\end{tabular}

Desta maneira, adicionando-se as novas heurísticas à lista de heurísticas originais, pode ser possível obter um resultado de avaliação heurística ainda melhor para a categoria de portais educacionais.

O caminho realizado neste trabalho $\mathrm{e}$ as conclusões dele decorrentes recomendam a utilização de heurísticas contextuais no método de avaliação heurística como instrumento de inspeção de usabilidade em interfaces de Portais Universitários.

\section{Conclusão}

O método de avaliação heurística utilizado para a inspeção do Portal da Unifesp demonstrou ser eficaz ao revelar problemas de usabilidade, além de ser barato, rápido e fácil.

O uso do novo conjunto de heurísticas específicas para Portais Universitários permitiu a descoberta de problemas de usabilidade não contemplados pelas heurísticas originais do Instituto Faber-Ludens. Sendo assim, futuras avaliações de Portais Universitários poderão utilizar as mesmas heurísticas utilizadas nesse trabalho e obter maior número de problemas de usabilidades específicos do domínio "Portal Educacional". As heurísticas utilizadas nessa avaliação podem ser utilizadas como um referencial, podendo sofrer alterações conforme as necessidades da equipe avaliadora ou o objetivo do trabalho.

Desta forma, propõe-se que em novas avaliações de portais educacionais sejam utilizadas o novo conjunto de heurística para detecção do maior número possível de problemas de usabilidade. Isto irá contribuir sobremaneira para a qualidade do produto final, uma vez que o método de avaliação heurística deve ser utilizado como parte do ciclo de desenvolvimento de softwares.

\section{Referências}

ALMSTROM, P. Usability Inspections. Affectus AB. Retrieved on February, v. 9, p. 2003, 2001.

AMSTEL, F. M. C. Heurísticas para Avaliação de Interfaces de Portais Universitários. Disponível em: <http://www.faberludens.com.br/pt-br/node/5564>. Acesso em: 10 maio. 2012. 
ANDRADE, A. Usabilidade de interfaces web: avaliação heurística no jornalismo on-line. [s.l.] Editora E-papers, 2007.

CASTRO SALGADO, L. C.; BIM, S. A.; DE SOUZA, C. S. Comparação entre os métodos de avaliação de base cognitiva e semiótica. Proceedings of VII Brazilian symposium on Human factors in computing systems. Anais...ACM, 2006

COELHO, ORLANDO BISACCHI, DANIEL MONEGATTO SANTORO. "A Sinergia entre as Heurísticas de Usabilidade de Software e as Heurísticas de EnsinoAprendizagem do ponto de vista da Educação à Distância Mediada pela Web." Anais do Simpósio Brasileiro de Informática na Educação. Vol. 1. No. 1. 2002.

CYBIS, W. DE A. et al. Uma abordagem ergonômica para o desenvolvimento de sistemas interativos. Anais do I Workshop sobre Fatores Humanos em sistemas computacionais: compreendendo usuários, construindo interfaces. Maringá-PR. Anais...1998

DAHER, L. A.; ELKABANI, I. Usability Evaluation of some Lebanese Universities Web Portals. 2012.

GONZÁLEZ, M. P. et al. Testing website usability in Spanish-speaking academia through heuristic evaluation and cognitive walkthroughs. Int J Univers Comp Sci, v. 14, n. 9, p. 1513-1527, 2008.

JEFFRIES, R. et al. User interface evaluation in the real world: a comparison of four techniques Proceedings of the SIGCHI conference on Human factors in computing systems: Reaching through technology. Anais...ACM, 1991

KOSTARAS, N.; XENOS, M. Assessing educational web-site usability using heuristic evaluation rulesProceedings of 11th Panhellenic Conference in Informatics. Anais...Hellenic Open University, School of Sciences \& Technology, Patras, Greece: 2007

NIELSEN, J. Usability inspection methodsConference companion on Human factors in computing systems. Anais...ACM, 1994

NIELSEN, J. Severity Ratings for Usability Problems: Article by Jakob Nielsen. Disponível em: <http://www.nngroup.com/articles/how-to-rate-the-severity-of-usabilityproblems/>. Acesso em: 24 mar. 2013.

NIELSEN, J. Designing web usability. [s.1.] Pearson Deutschland GmbH, 2000.

NIELSEN, J.; HACKOS, J. T. Usability engineering. [s.l.] Academic press San Diego, 1993. v. 125184069

NIELSEN, J.; MOLICH, R. Heuristic evaluation of user interfacesProceedings of the SIGCHI conference on Human factors in computing systems: Empowering people. Anais...ACM, 1990

NORMAN, D. A.; DRAPER, S. W. User centered system design; new perspectives on human-computer interaction. [s.l.] L. Erlbaum Associates Inc., 1986.

ROCHA, H. V.; BARANAUSKAS, M. C. C. Design e avaliação de interfaces humanocomputador. [s.l.] Unicamp, 2003.

SANTINHO, M. Avaliação Heurística e testes com utilizadores: dois métodos, dois resultados. Disponível na Internet na URL:<www. simplicidade. com>. Acesso em set, 2001.

SHNEIDERMAN, B. Designing the user interface. [s.1.] Pearson Education India, 1998.

STEWART, K. Notable - Better Interfaces Through Faster Iterations. [s.l: s.n.]. 